\title{
BLIND SEPARATION OF CONVOLUTIVE MIXTURES BASED ON SECOND ORDER AND THIRD ORDER STATISTICS
}

\author{
Zhongfu Ye ${ }^{l}$, Chunqi Chang ${ }^{2}$, Chen Wang ${ }^{l}$, Jian Zhao ${ }^{I}$, and Francis H. Y. Chan ${ }^{2}$ \\ 1. Institute of Statistical Signal Processing, University of Science and Technology of China \\ Hefei, Anhui Province, P.R. China \\ 2. Department of Electrical and Electronic Engineering, The University of Hong Kong \\ Pokfulam Road, Hong Kong, Hong Kong SAR, P.R. China \\ E-mail: yezf@ustc.edu.cn, cqchang@eee.hku.hk, fhychan@eee.hku.hk
}

\begin{abstract}
This paper addresses the problem of blind separation of linear convolutive mixtures. We first reformulate the problem into a blind separation of linear instantaneous mixtures, and then a statistical approach is applied to solve the reformulated problem. From the statistics of the mixtures, two kinds of matrix pencils are constructed to estimate the mixing matrix. The original sources are then separated with the estimated mixing matrix. For the purpose of computational efficiency and robustness, in the matrix pencil, one matrix is constructed from the second order statistics, and the other is constructed from the third order statistics. The proposed novel methods do not require the exact knowledge of the channel order. Simulation results show that the methods are robust and have good performance.
\end{abstract}

\section{INTRODUCTION}

Blind separation of convolutive mixtures has attracted much attention recently, for both theoretical development and practical applications [1-6]. The problem of blind separation of convolutive mixtures is also known as blind multi-channel deconvolution or blind multi-channel equalization. This technique, when well developed, can have many practical applications in various areas such as wireless communication, radar, sonar, speech recognition, echo cancellation, and so on. Blind separation of convolutive mixtures has two essential problems to be solved jointly. One is blind signal separation, and the other is blind deconvolution. Blind signal separation was proposed from the viewpoint of signal processing in the middle 1980s, and was later developed into the concept of independent component analysis (ICA), which has a close relationship with an rather older concept, factor analysis, in the discipline of statistics. Interestingly, it has been shown that the two problems, blind signal separation of instantaneous mixtures and single channel blind deconvolution, are closely related [6-8]. Therefore, in the literature, many approaches to blind separation of convolutive mixtures are based on either blind separation of instantaneous mixtures or single channel blind deconvolution. For details and references, the reader is referred to [6-9]. In this paper, our approach to blind separation of convolutive mixtures will be based on the methodology of reformulating the problem to blind separation of instantaneous mixtures.

Though it has been suggested in [6-8] that convolutive model can be reformulated into instantaneous model and thus classic methods for blind instantaneous source separation can be directly used to solve the problem of blind convolutive source separation, in the literature there are not too many works investigating this methodology on its validity, performance, and special problems involved. Indeed, the resulting high-dimensional instantaneous model has a very special structure of its mixing matrix, and this special structure may make the problem difficult and complicated by inducing rank deficiency of the mixing matrix. Recently, this methodology was investigated in [4] using a second order statistical approach and in [2] using a fourth order statistical approach.

In this paper, we propose a matrix pencil approach by jointly using second order and third order statistics, and extend our earlier results in $[10,11]$ to the general case with convolutive mixtures. Our major concern is on computational efficiency and robustness. For this purpose we use lower order statistics, that is, second order statistics and third order statistics. For higher order statistics, only third order statistics will be used, because the computation efficiency and robustness of the statistics with order higher than three will be degraded comparing to their counterparts of third order statistics. For using third order statistics, the signals need to be asymmetric in their statistical distributions. Asymmetrical distribution is common in many practical applications, but not in QAM 
communication. However, the treatments used in [12-13] can be adopted for applying our method to the area of communication.

\section{PROBLEM STATEMENT}

For simplicity in derivation, we consider a noiseless convolutive mixing model with $\mathrm{M}$ sources and $\mathrm{N}$ mixtures, as follows:

$\left[\begin{array}{c}x_{1}(t) \\ \vdots \\ x_{N}(t)\end{array}\right]=\mathbf{x}(t)=\mathbf{A}(t) * \mathbf{s}(t)=\left[\begin{array}{ccc}a_{11}(t) & \cdots & a_{1 M}(t) \\ \vdots & \ddots & \vdots \\ a_{N 1}(t) & \cdots & a_{N M}(t)\end{array}\right] *\left[\begin{array}{c}s_{1}(t) \\ \vdots \\ s_{M}(t)\end{array}\right]$

where "**" denotes the operator of convolution. Assume that the filters $a_{i j}(t)$ are FIR with maximal length as L, then we have

$$
x_{i}(t)=\sum_{j=1}^{M} \sum_{i=0}^{L-1} a_{i j}(l) s_{j}(t-l) \text { for } i=1, \cdots, N
$$

Stacking the mixtures $\mathrm{K}$ times, we have the reformulated instantaneous mixing model:

$$
\left[\begin{array}{c}
\mathbf{x}_{1}(t) \\
\vdots \\
\mathbf{x}_{N}(t)
\end{array}\right]=\tilde{\mathbf{x}}(t)=\tilde{\mathbf{A}} \tilde{\mathbf{s}}(t)=\left[\begin{array}{ccc}
\mathbf{A}_{11} & \cdots & \mathbf{A}_{1 M} \\
\vdots & \ddots & \vdots \\
\mathbf{A}_{N 1} & \cdots & \mathbf{A}_{N M}
\end{array}\right]\left[\begin{array}{c}
\mathbf{s}_{1}(t) \\
\vdots \\
\mathbf{s}_{M}(t)
\end{array}\right]
$$

where

$$
\begin{gathered}
\mathbf{x}_{i}(t)=\left[\begin{array}{llll}
x_{i}(t) & \cdots & x_{i}(t-K+1)
\end{array}\right]^{T} \\
\mathbf{s}_{j}(t)=\left[\begin{array}{llll}
s_{j}(t) & \cdots & s_{j}(t-L-K+2)
\end{array}\right]^{T} \\
\mathbf{A}_{i j}=\left[\begin{array}{ccccc}
a_{i j}(0) & \cdots & a_{i j}(L-1) & \cdots & 0 \\
\vdots & \ddots & \ddots & \ddots & \vdots \\
0 & \cdots & a_{i j}(0) & \cdots & a_{i j}(L-1)
\end{array}\right]
\end{gathered}
$$

Note that the dimension of $\mathbf{A}_{i j}$ is $K \times(K+L-1)$, and the dimension of $\tilde{\mathbf{A}}$ is $N K \times M(K+L-1)$. For (3) to be solvable, we need the following assumptions:

A1) Components in $\tilde{\mathbf{s}}(t)$ are mutually independent.

A2) $\overline{\mathbf{A}}$ has full column rank.

Equivalently, we need the original convolutive system:

1. Each source signal $s_{j}(t)$ is i.i.d.

2. Sources are mutually independent.

3. More mixtures than sources for nontrivial cases, i.e., $N>M$ for $L>1$.

4. There is no common zero among the convoluting filters.

Points 1 and 2 are due to $A 1$, while points 3 and 4 are due to $A 2$. These requirements are commonly used in higher order statistics based approaches to blind separation of convolutive mixtures.

\section{ALGORITHMS}

Due to the assumptions in section 2, higher order statistics is needed to solve the blind separation problem (1) or (3). Here we assume that third order statistics exists for all the source signals. Due to the property of cumulant [14], define a third order cumulant matrix as

$$
\begin{aligned}
& \mathbf{C}_{i}(k)=\operatorname{cum}_{3}\left\{\tilde{\mathbf{x}}(t) \dot{\mathbf{x}}^{T}(t) x_{i}(t-k)\right\} \\
& =\tilde{\mathbf{A}} \operatorname{cum}_{3}\left\{\tilde{\mathbf{s}}(t) \tilde{\mathbf{s}}^{T}(t) \sum_{i=0}^{L-1} a_{i j}(l) s_{j}(t-k-l)\right\} \tilde{\mathbf{A}} \\
& =\tilde{\mathbf{A}} \operatorname{diag}\left\{\mathbf{D}_{1}(k), \cdots, \mathbf{D}_{M}(k)\right\} \tilde{\mathbf{A}}^{T} \\
& =\tilde{\mathbf{A}} \Lambda_{i}(k) \tilde{\mathbf{A}}^{T}
\end{aligned}
$$

where

\subsection{Matrix pencil method based on cumulant matrix}

Arbitrarily select two indices $i_{1}$ and $i_{2}$, we can formulate a matrix pencil

$$
\left\{\mathbf{C}_{i_{1}}(k), \mathbf{C}_{i_{2}}(k)\right\}=\left\{\mathbf{A} \Lambda_{i_{1}}(k) \tilde{\mathbf{A}}^{T}, \mathbf{A} \Lambda_{i_{2}}(k) \tilde{\mathbf{A}}^{T}\right\}
$$

Apply ESPRIT [10] or generalized eigenvalue decomposition [10] on this matrix pencil, then we get $L$ columns (column $k+I$ to column $k+L$ ) of $\mathbf{A}_{i j}$ for each pair $i j$, i.e.,

$$
\breve{\mathbf{A}}_{i j}=\left[\begin{array}{ccccc}
\vdots & \vdots & \ddots & \vdots & \vdots \\
a_{i j}(1) & a_{i j}(2) & \cdots & a_{i j}(L-1) & 0 \\
a_{i j}(0) & a_{i j}(1) & \cdots & a_{i j}(L-2) & a_{i j}(L-1) \\
0 & a_{i j}(0) & \cdots & a_{i j}(L-3) & a_{i j}(L-2) \\
\vdots & \vdots & \ddots & \vdots & \vdots
\end{array}\right] \leftarrow k+1
$$

From $\breve{\mathbf{A}}_{i j}$ we can get $a_{i j}(l), l=0, \cdots, L-1$, and then we can construct $\mathbf{A}_{i j}$ based on (4), and at last we get the mixing matrix $\tilde{\mathbf{A}}$. With $\tilde{\mathbf{A}}$ available, the source signal vector, $\mathbf{s}(t)$, can be filtered through the mixtures, $\mathbf{x}(t)$, by standard methods.

To improve the computational efficiency and robustness of the algorithm, we can replace one of the cumulant matrices in the matrix pencil by the second order cumulant matrix, i.e., the following correlation matrix $\mathbf{R}_{\tilde{x}}=\operatorname{cum}_{2}\left\{\tilde{\mathbf{x}}(t) \tilde{\mathbf{x}}^{T}(t)\right\}=\mathbf{A} \operatorname{cum}_{2}\left\{\mathbf{s}(t) \overline{\mathbf{s}}^{T}(t)\right\} \tilde{\mathbf{A}}^{T}=\mathbf{A R}_{\dot{s}} \tilde{\mathbf{A}}^{T}$ Simulation results show that using $\mathbf{R}_{\tilde{x}}$ will improve the performance. In the simulation section we will use the algorithm based on $\left\{\mathbf{C}_{i}(k), \mathbf{R}_{\dot{x}}\right\}$. 
3.2 Matrix pencil method based on weighted summation of cumulant matrices

To improve the robustness of the algorithm, we formulate a weighted summation of the above defined cumulant matrices, as follows:

$$
\begin{aligned}
\mathbf{C}_{i} & =\sum_{k=0}^{K-1} w_{k} \mathbf{C}_{i}(k) \\
& =\operatorname{cum}_{3}\left\{\tilde{\mathbf{x}}(t) \tilde{\mathbf{x}}^{T}(t) \sum_{k=0}^{K-1} w_{k} x_{i}(t-k)\right\} \\
& =\tilde{\mathbf{A}} \operatorname{diag}\left\{\mathbf{D}_{1} \quad \cdots \quad \mathbf{D}_{M}\right\} \tilde{\mathbf{A}}^{T} \\
& =\mathbf{A} \Lambda_{i} \tilde{\mathbf{A}}^{T}
\end{aligned}
$$

where $\mathbf{D}_{j}=\sum_{k=0}^{K-1} w_{k} \mathbf{D}_{j}(k), \Lambda_{i}=\sum_{k=0}^{K-1} w_{k} \Lambda_{i}(k)$, and $\left\{w_{k}\right\}$ are randomly selected from $[0,1]$.

Then we can formulate a matrix pencil $\left\{\mathbf{C}_{i_{1}}, \mathbf{C}_{i_{2}}\right\}$ or $\left\{\mathbf{C}_{i}, \mathbf{R}_{\dot{x}}\right\}$, and apply the same approach as in section 3.2 .

\section{NUMERICAL SIMULATIONS}

Numerical experiments are carried out in the MATLAB environment to evaluate the performance of the developed algorithms. We consider a system with parameters as $M=2, N=3$, and $L=2$. The two random source signals have exponent distribution with the parameter equal to 3 . The multi-channel FIR system, which represents the convolutive mixing matrix, is described as

$\mathbf{a}_{11}=[-1.18441 .8042], \mathbf{a}_{12}=[0.2255-1.8634]$,

$\mathrm{a}_{21}=[0.6813-0.8221], \mathrm{a}_{22}=[-0.5328-0.3542]$.

Let $K=2$, the mixing matrix can be written as

$\left[\begin{array}{cccccc}-1.1844 & 1.8042 & 0 & 0.2255 & -1.8634 & 0 \\ 0 & -1.1844 & 1.8042 & 0 & 0.2255 & -1.8634 \\ 0.6813 & -0.8221 & 0 & 0.5328 & -0.3542 & 0 \\ 0 & 0.6813 & -0.8221 & 0 & 0.5328 & -0.3542 \\ -0.5626 & -0.6966 & 0 & 0.3565 & -0.4997 & 0 \\ 0 & -0.5626 & -0.6966 & 0 & 0.3565 & -0.4997\end{array}\right]$

The algorithms described in Section 3.1 and Section 3.2 have been applied to solve the blind separation problem. In both algorithms, the correlation matrix $\mathbf{R}_{\dot{\varepsilon}}$ is used in the matrix pencil. Two types of experiments have been done to demonstrate the performance of the two algorithms. In one experiment, different numbers of data points are used to evaluate the performance, while the signal to noise ratio (SNR) is fixed. In the other experiment, various SNR are used with fixed number of data points. Results are shown in Figure 1 to Figure 4. It is demonstrated in the figures that the performance of the algorithm in section 3.2 is better than that of the algorithm in section 3.1, especially when the number of data points is relatively small. When a large number of data points is adopted, the two algorithms have similar performance.

\section{CONCLUTIONS}

In this paper, the problem of blind separation of convolutive mixed mixtures is considered. Novel methods based on second order statistics and third order statistics are developed to solve this problem. The approach in this paper reformulate the convolutive mixing system to an equivalent instantaneous mixing system, and then matrix pencils are formulated based on second order and third order cumulant matrices. Numerical simulations show that the methods have good performance. Further improvement of the methods, and performance comparison with other approaches, will be studied later.

\section{REFERENCES}

[1] L.C. Parra and C.V. Alvino, "Geometric source separation: merging convolutive source separation with geometric beamforming," IEEE Trans. Speech and Audio Processing, vol.10, no.6, pp.352-262, Sep. 2002.

[2] J. Liang and Z. Ding, "A simple cumulant based approach for multiuser channel identification," Proc. ISCAS2002, vol.3, pp.659-662, 2002.

[3] J.K. Tugnait and B. Huang, "Blind estimation and equalization of MIMO channels via multidelay whitening," IEEE Journal on Selected Areas in Communication, vol.19, no.8, pp.1507-1519, Aug. 2001.

[4] H. Bousbia-Salah, A. Belouchrani, and K. Abed-Meraim, "Blind separation of convolutive mixtures using joint block diagonalization," Proc. Int. Symp., Signal Processing and its Applications (ISSPA), Kuala Lumpur, Malaysia, pp.1316, Aug. 2001.

[5] C.T. Ma, Z. Ding, and S.F. Yau, "A two-stage algorithm for MIMO blind deconvolution of nonstationary colored signals," IEEE Trans. Signal Processing, vol.48, no.4, pp. 1187-1192, Apr. 2000.

[6] C. Chang, Blind Signal Estimation Using Second Order Statistics, PhD thesis, The University of Hong Kong, 2000.

[7] S. Haykin (Ed), Unsupervised Adaptive Filtering, John Wiley \& Sons, 2000.

[8] A. Hyvarinen, J. Karhunen, and E. Oja, Independent Component Analysis, John Wiley \& Sons, 2001.

[9] A. Cichocki and S. Amari, Adaptive Blind Signal and Image Processing: Learning algorithms and Applications, John Wiley \& Sons, 2002.

[10] C. Chang, Z. Ding, S. F. Yau, and F.H.Y. Chan, "A matrixpencil approach to blind separation of colored nonstationary signals," IEEE Trans. Signal Processing, vol.48, no.3, pp.900-907, Mar. 2000.

[11] Z. Ye, P.C.Ching, K.M.Wong, "A Novel Technique for the Blind Estimation of a Channel Matrix," Proceedings of the IEEE International Conference on Electronics, Circuits, and Systems, pp.441-444, 1998.

[12] K.I. Chung, and C.T. Lim, "Transform methods of PAM signals for asymmetric distribution in 3rd-order blind equalizer," Proc IEEE TECON96, vol.2, pp.614-618, 1996. 
[13] C.D. Murphy, "Third-order blind equalization properties of hexagonal constellation," Proc. of $10^{\text {th }}$ IEEE Workshop on Statistical Signal and Array Processing, pp.697-701, 2000.

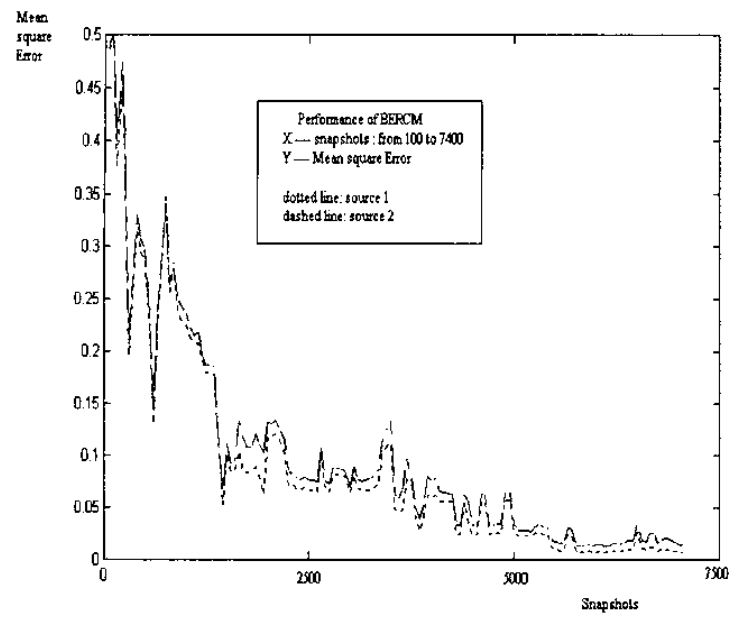

Figure 1. Performance of the algorithm described in section 3.1 , where the SNR is fixed, but the number of data points varies from 100 to 7400 .

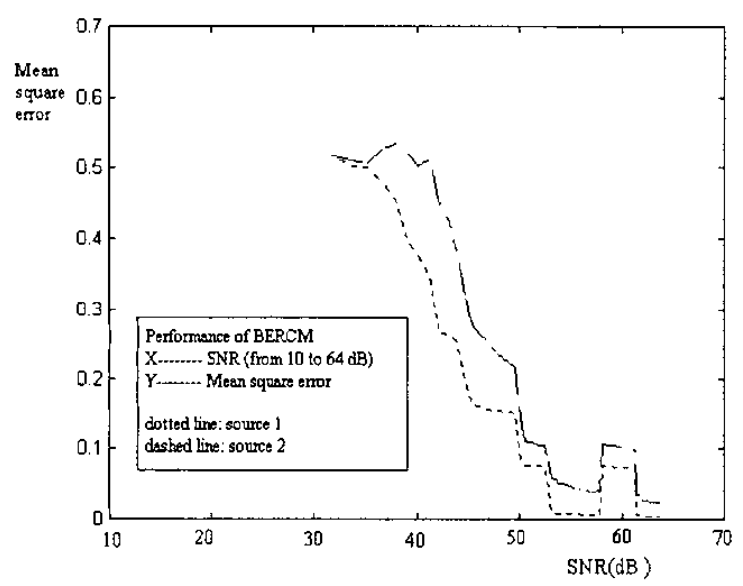

Figure 2. Performance of the algorithm described in section 3.1, where the number of data points is fixed, but the SNR varies from $10 \mathrm{~dB}$ to $64 \mathrm{~dB}$.
[14] C.L. Nikias and A.P. Petropulu, Higher-order spectra analysis: a nonlinear signal processing framework, Prentice-Hall, New Jersey, 1993.

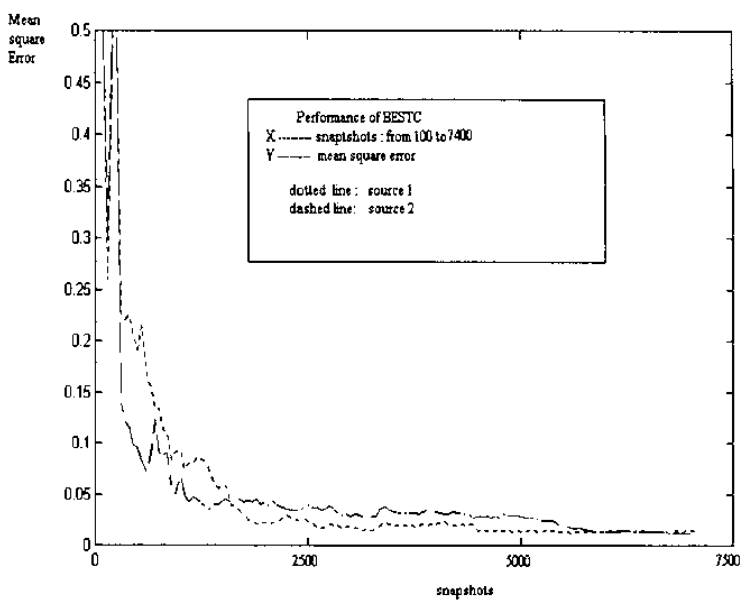

Figure 3. Performance of the algorithm described in section 3.2, where the SNR is fixed, but the number of data points varies from 100 to 7400 .

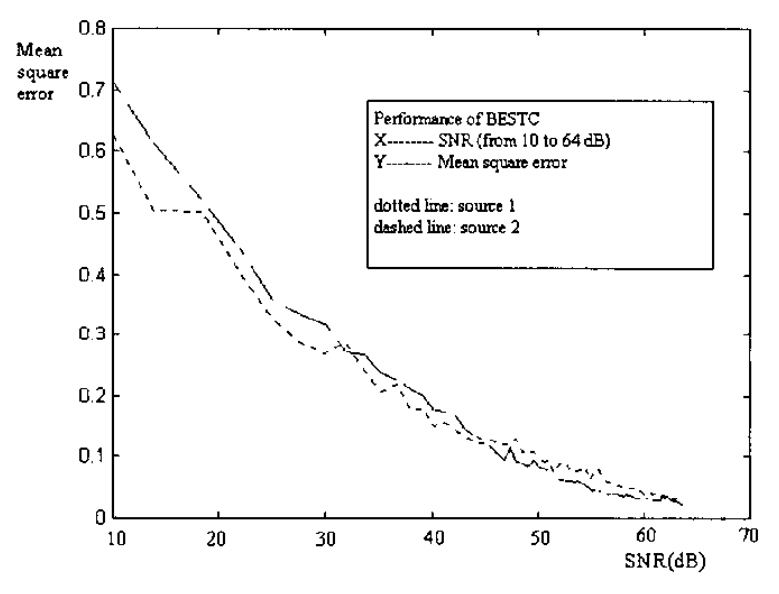

Figure 4. Performance of the algorithm described in section 3.2, where the number of data points is fixed, but the SNR varies from $10 \mathrm{~dB}$ to $64 \mathrm{~dB}$. 